\title{
Acknowledgment of Reviewers
}

Ron Acierno

James Russell Andretta

Timothy Apodaca

Jeff Aspelmeier

Jaedon Avey

Tammy Ayres

Cathy Bell

Teri Browne

Jessica Burris

Denise Chavira

Kathleen Contrino

Annette S. Crisanti

Lisa Curtin

Laura DeHaan

Louise Dixon*

Julie Ebin

Ellen Fischer

Lara Gerassi

Michael Glasser

Jennifer Green

Daniel Gros

Judith Hahn

Jameson K. Hirsch

\section{Alysia Hoover-
Lisa Horowitz}

J. P. Jameson

Jordan Joyner*

Laura King

Aimee Kliewer*

Julie Mae Koch

Celine Larkin

Alexander Latham*

Valerie Leake

Jennifer Dunbar

Lenardson

David Leon

Peter Lundqvist

Shannon Lynch

David Mandich*

Erick C. Messler

Kurt Michael

Shannon Monnat

Dermot O'Reilly

Elizabeth Perkins

Thomas Pierce

Gary Potter
Gaberiella Puleo*

Sara Quandt

Randy Quevillon

Marcela Raffaelli

Thaddeus Reece*

Jana Rostocki*

Kai Schafft

Sharon Seiling

Lisa Shannon

Claire Snell-Rood

Justin Sprung

Jennifer Stroup

Jean A. Talbot

Dawn Trussell

Daniel Walinsky

Tara Warner

Teddy Warner

Carl Weems

Lisa Wexler

Barbara Wilder

Elizabeth Williams

Alese Wooditch

Peter Yellowlees 